\title{
多晶硅表面金属催化化学腐蚀法制线研究现状
}

\author{
武晓玮 ${ }^{1,2}$, 李佳艳 ${ }^{1,2}$ \\ (大连理工大学 1. 三束材料改性教育部重点实验室; 2. 材料科学与工程学院, 大连 116024)
}

摘 要: 在多晶硅太阳能电池的生产过程中, 金刚线切割(Diamond wire sawing, DWS)技术具有切割速度快、精度高、 原材料损耗少等优点, 受到了广泛关注。金刚线切割多晶硅表面形成的损伤层较浅, 与传统的酸腐蚀制线技术无法 匹配, 金属催化化学腐蚀法应运而生。金属催化化学腐蚀法制线具有操作简单、结构可控且易形成高深宽比的线面 等优点, 具有广阔的应用前景。本文总结了不同类型的金属催化剂在制线过程中的腐蚀机理及其形成的线面结构, 深入分析和讨论了具有代表性的银、铜的单一及复合催化腐蚀过程及线面结构和电池片性能。最后对金刚线切割 多晶硅片表面的金属催化化学腐蚀法存在的问题进行了分析, 并展望了未来的研究方向。

关 键 词: 金刚线切割; 多晶硅; 金属催化化学腐蚀法; 制线; 综述

中图分类号: TM914 文献标志码: A

\section{Texturing Technology on Multicrystalline Silicon Wafer by Metal-catalyzed Chemical Etching: a Review}

\begin{abstract}
WU Xiaowei ${ }^{1,2}$, LI Jiayan ${ }^{1,2}$
(1. Key Laboratory of Materials Modification by Laser, Ion, and Electron Beams, Dalian University of Technology, Dalian 116024, China; 2. School of Materials Science and Engineering, Dalian University of Technology, Dalian 116024, China)

Abstract: In the production process of multicrystalline silicon solar cells, diamond wire sawing (DWS) technology attracts wide attention because of its advantages of high cutting speed, high precision and less loss of raw materials. But the traditional acid etching technology cannot match the shallow damage layer formed on the surface of diamond wire sawn multicrystalline silicon wafer to make the texture surface. On the contrary, the metal-catalyzed chemical etching method owns the advantages of simple operation, controllable structure, and being easy to form the structure with high aspect ratio, indicating a wide range of application on diamond wire sawn multicrystalline silicon wafer. This paper systematically summarizes the work of the etching mechanisms and the structures of textures by different metal catalysts in the process of making texture surface, and deeply discusses the single and composite catalytic etching process of $\mathrm{Ag}$ and $\mathrm{Cu}$, the structure of texture surface, and the performance of solar cells. Finally, the problems of metal-catalyzed chemical etching on the surface of diamond wire sawn multicrystalline silicon are analyzed, and their future research directions are prospected.
\end{abstract}

Key words: diamond wire sawing; multicrystalline silicon; metal-catalyzed chemical etching; texturization; review

收稿日期: 2020-06-30; 收到修改稿日期：2020-08-30; 网络出版日期：2020-10-23

基金项目: 国家自然科学基金(51574057) National Natural Science Foundation of China (51574057)

作者简介: 武晓玮(1991-), 男, 博士研究生. E-mail: wuxiaowei261@163.com WU Xiaowei(1991-), male, PhD candidate. E-mail: wuxiaowei261@163.com

通信作者: 李佳艳, 副教授. E-mail: lijiayan@dlut.edu.cn LI Jiayan, associate professor. E-mail: lijiayan@dlut.edu.cn 
太阳能作为一种新型的可再生能源，是目前最 清洁和环保的能源之一，具有储量巨大、来源稳定、 清洁无污染等优点 ${ }^{[1]}$ 。光伏发电是太阳能能源利用 的主要形式 ${ }^{[2]}$,预计在 2050 年和 2100 年, 将分别占 全球总发电量的 20\%和 70\%, 已成为 21 世纪最重要 的新能源之一 ${ }^{[3]}$ 。

太阳能电池种类繁多, 结构多样, 光电转换效 率也在逐年提高。在众多太阳能电池中, 晶体硅太 阳能电池以其生产工艺成熟、稳定性好、原材料来 源广、储量大等优势，在光伏产业中一直占据 $90 \%$ 以上的份额 ${ }^{[4]}$ 。作为晶体硅太阳能电池的主要原料, 单晶硅的拉棒工艺复杂, 制备成本较高; 而多晶硅 (mc-Si)的方形铸锭工艺简单, 硅料利用率较高, 生 产成本低, 优势显著 ${ }^{[5]}$ 。

从多晶硅铸锭到多晶硅片, 切割技术是降低生 产成本、提高硅片出成率的关键因素。多线切割可 以将晶体硅的铸锭切割成一定厚度的硅片 ${ }^{[6-7]}$, 传 统的主要切割方式为砂浆切割 (Multi-wire slurrly sawing, MWSS), 砂浆由高硬度的 SiC 颗粒磨料和 以聚乙二醇为主、其他添加剂为辅的切割液组成, 切割时利用钢丝带动砂浆中的碳化硅颗粒对硅片进 行挤压磨削切割。砂浆切割技术是光伏产业中应用 最广泛、最成熟的技术, 但存在易引入杂质、废料 回收困难、硅的损耗较大、效率低等缺点 ${ }^{[8]}$ 。近年 来新兴的金刚线切割(DWS)技术是利用电镀或树脂 粘结的方法，将高硬度、耐磨的金刚石颗粒固定在 不锈钢丝上制成固结磨料锯丝，通过线锯和金刚石 颗粒的共同运动对硅片进行切割。具有速度快、精 度高、废液易回收、对环境污染小等显著优点 ${ }^{[9]}$, 已 经全面取代砂浆切割成为制备多晶硅片的主流技 术。图 1 为两种切割方法的原理示意图。

\section{1 金刚线切割多晶硅片制线}

硅片制线是对切割后的硅片进行化学处理, 在 晶体硅的表面形成相应的微观结构, 使太阳光入射 到晶体硅表面后发生多次反射，从而降低晶体硅太
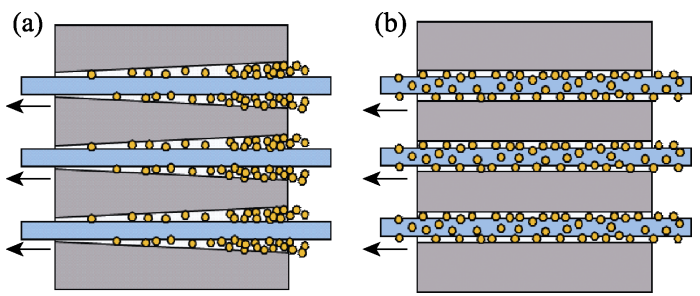

图 1 (a)砂浆切割和(b)金刚线切割示意图

Fig. 1 Schematic diagram of (a) MWSS technique and (b) DWS technique
阳能电池对入射光的反射率, 最终提高光电转换 效率 ${ }^{[10]}$ 。

多晶硅的线面结构需要满足以下三个要求。 1)结构分布均匀, 确保多晶硅片表面对入射光的吸 收程度一致。这样经扩散、镀膜、丝网印刷等后续 工艺形成的太阳能电池片性能较稳定。2)合适的深 宽比，这样既有利于增强光陷阱效应、降低硅片反 射率，还可以减小微结构的表面积、抑制载流子的 复合、提高光电转换效率。3)较好的微观结构。线 切割及制线过程会给硅片表面带来裂纹、晶格畸变 等损伤，裂纹面上有大量硅原子的悬挂键，易造成 载流子的复合，而晶格畸变对载流子有严重的散射 作用，从而影响迁移率。

图 2 为 MWSS 和 DWS 两种方法切割的多晶硅 片表面扫描电子显微镜(SEM)照片。可以看出, 砂浆 切割的多晶硅片表面粗䊁, 在切割过程中 $\mathrm{SiC}$ 颗粒 和硅片表面相互作用留下了大量分布均匀的凹坑。 而金刚线切割的多晶硅片表面相对平滑，在相互平 行的切割纹之间有随机分布的破碎凹坑。这是由于 在切割过程中, 切割线上的金刚石颗粒对晶体硅压 力较大, 颗粒以脆性崩脱的方式切割硅, 进而形成 破碎的凹坑 ${ }^{[11-12]}$ 。Bidiville 等 ${ }^{[13]}$ 利用扫描电子显微 镜和拉曼光谱对金刚线切割多晶硅片表面进行表 征, 发现其表面存在由强烈的塑性变形造成的非 晶层, 对硅片的腐蚀有抑制作用。上述两种多晶硅 片表面性状的巨大差异，导致化学处理效果存在 明显的区别。针对砂浆切割硅片的传统制线工艺无 法直接用于金刚线切割多晶硅片中, 因此, 开发适 合于金刚线切割多晶硅片的制线工艺成为近期的 研究热点。
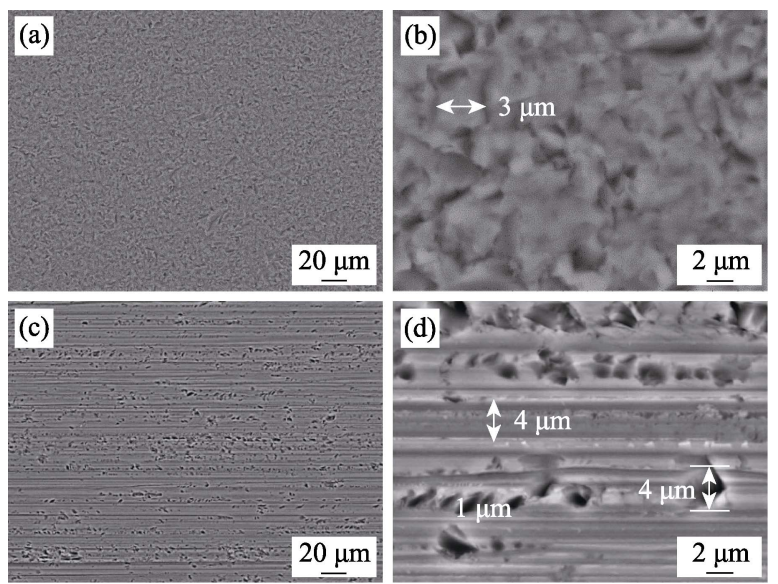

图 2 (a, b)砂浆切割和(c, d)金刚线切割后多晶硅片表面形 貌 SEM 照片

Fig. 2 SEM images of (a, b) MWSS and (c, d) DWS multicrystalline silicon wafer surface 


\section{1 制线方法}

目前国内外针对金刚线切割多晶硅片的制线方 法主要包括以下几种: 添加剂改性酸腐蚀法 ${ }^{[14]}$ 、电 化学腐蚀法 ${ }^{[15]} 、 气$ 相刻蚀法 ${ }^{[16]}$ 、反应离子刻蚀法 (Reaction ion etching, RIE) ${ }^{[17]}$ 和金属催化化学腐蚀 法(Metal-catalyzed chemical etching, MCCE $)^{[18-19]}$ 等。

传统酸腐蚀法是针对砂浆切割多晶硅片的制线 方法。图 3 为采用酸腐蚀法在金刚线切割多晶硅片 表面制线后的表面 SEM 形貌和反射率曲线。从图中 可知, 传统酸腐蚀法制线后线面结构受划痕影响较 大, 腐蚀坑均沿划痕方向分布, 且均匀性较差。反射 率很高, 约为 $36.72 \%$, 无法达到太阳能电池对多晶 硅片表面反射率的要求。为了降低硅片表面反射率, 在腐蚀液中引入添加剂, 改变腐蚀反应过程, 从而 优化多晶硅表面形貌, 可以在一定程度内降低反射 率, 但仍然无法达到要求。

气相刻蚀法的温度场较难控制, 稳定性、均匀 性较差, 且酸蒸汽会对设备造成腐蚀, 易引起环境 污染等问题。反应离子刻蚀法工艺复杂、设备昂贵, 无法大规模应用。相比之下, MCCE 具有以下显著 优点: 1)操作简单、成本低; 2)线面形貌易控制, 结构 稳定；3)易获得高深宽比的结构, 减反射性能优异; 4)可以制备各种不同尺寸的线面结构。因此 MCCE 适合大规模生产，具有广阔的应用前景。

\section{2 金属催化化学腐蚀法制线}

MCCE 方法由 Dimova-Malinovska 团队 ${ }^{[20]}$ 于 1997 年首先报道, 他们通过 $\mathrm{HF}$ 和 $\mathrm{HNO}_{3}$ 的水溶液 对硅基体上的铝进行腐蚀而形成多孔硅。随后 $\mathrm{Li}$ 和 Bohn ${ }^{[21]}$ 对 MCCE 法进行了详细的研究。

MCCE 法通常会在硅片表面沉积一层 Ag、Cu、

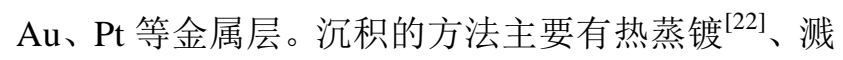
射 $^{[23]} 、$ 化学沉积 ${ }^{[24]}$ 、聚焦离子束辅助沉积 ${ }^{[25]}$ 等。将 沉积金属后的硅片放入含有 HF 和氧化剂的溶液中

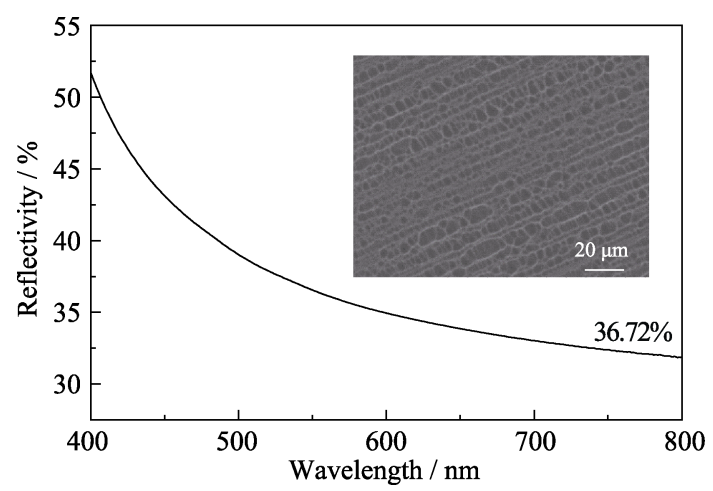

图 3 酸腐蚀法制线后硅片表面 SEM 形貌和反射率曲线

Fig. 3 SEM morphology and reflectivity for texture surface of silicon wafer by acid etching
进行腐蚀, 其中最常用的氧化剂为双氧水。图 4 为 MCCE 法的反应原理示意图。

在金属的催化作用下，氧化剂优先在金属表面 发生反应, 产生大量空穴, 扩散到达金属和硅接触 的界面处, 硅被氧化形成 $\mathrm{SiO}_{2}$, 随即被溶液中的 $\mathrm{HF}$ 溶解。在反应过程中, 由于金属和硅界面处空穴浓 度较高, 因此在该界面处发生腐蚀的速率要远高于 没有金属覆盖的位置, 从而形成孔或凹坑状结构。 当空穴的注入速率超过 $\mathrm{HF}$ 溶解 $\mathrm{SiO}_{2}$ 的速率时, 界 面将会累积大量空穴, 并扩散到其它没有金属覆盖 的位置, 形成微多孔硅结构。在 MCCE 过程中, 反 应溶液与腐蚀产物均沿着金属和硅接触的界面传 递。总的反应式如(1)所示 ${ }^{[18]}$ :

$$
\mathrm{Si}+\frac{n}{2} \mathrm{H}_{2} \mathrm{O}_{2}+6 \mathrm{HF} \rightarrow n \mathrm{H}_{2} \mathrm{O}+\mathrm{H}_{2} \mathrm{SiF}_{6}+\frac{4-n}{2} \mathrm{H}_{2} \uparrow
$$

\section{2 不同类型金属的催化化学腐蚀}

在金刚线切割多晶硅片的金属催化化学腐蚀法 中, 最为常用的金属主要有 $\mathrm{Ag} 、 \mathrm{Cu}$ 的单一或复合 组元催化剂。下文将对不同类型催化剂的 MCCE 法 制线研究进展进行介绍。

\section{$2.1 \mathrm{Ag}$ 催化化学腐蚀}

$\mathrm{Ag}$ 作为一种贵金属, 被广泛应用于 MCCE 法 制线过程中。常用的腐蚀液体系为 $\mathrm{HF}$ 和 $\mathrm{AgNO}_{3}$ 的 混合溶液 $26-29]$ 。这是由于 $\mathrm{Ag}^{+} / \mathrm{Ag}$ 的电化学电位高 于硅祄底的费米能级, 硅片浸入到含有 $\mathrm{HF}$ 和 $\mathrm{AgNO}_{3}$ 的混合溶液中, 形成原电池。空穴可以从 $\mathrm{Ag}^{+}$ 中注入到硅的价带, $\mathrm{Ag}^{+}$被还原为 $\mathrm{Ag}$ 单质颗粒 ${ }^{[30]}$ 。由 $\mathrm{Ag}^{+}$注入到硅中的空穴使硅发生局部的氧化反应, 生成的 $\mathrm{SiO}_{2}$ 被溶液中的 $\mathrm{HF}$ 溶解。随着 $\mathrm{Ag}$ 颗粒下 方的硅被不断氧化、溶解, 硅表面形成腐蚀孔, Ag 颗粒沉积在被腐蚀的硅的孔洞中。在腐蚀前沿, 即 硅和沉积的 Ag 颗粒之间的界面, 优先发生电荷转 移。在 $\mathrm{Ag}^{+}$不断沉积的过程中, $\mathrm{Ag}$ 颗粒生长为枝晶 状结构。同时, 通过改变 $\mathrm{HF}$ 和 $\mathrm{AgNO}_{3}$ 的浓度, 可 以有效控制腐蚀速率以及 $\mathrm{Ag}$ 颗粒的形貌, 制备出

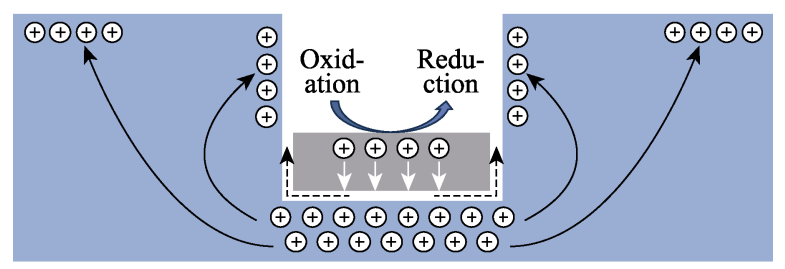

$\oplus$ Hole $\quad$ Si substrate $\quad$ Noble metal

图 4 MCCE 法原理示意图 ${ }^{[18]}$

Fig. 4 Schematic diagram of $\mathrm{MCCE}^{[18]}$ 
不同尺寸和结构的线面。

Zhuang 等 ${ }^{[31]}$ 基于 MCCE 法研究了一种可以消 除划痕获得理想线面的技术。将硅片置于 MCCE 溶 液 $\left(2.6 \mathrm{~mol} / \mathrm{L} \mathrm{HF} 、 1.1 \mathrm{~mol} / \mathrm{L} \mathrm{H}_{2} \mathrm{O}_{2}\right.$ 和 $2 \times 10^{-4} \mathrm{~mol} / \mathrm{L}$ $\left.\mathrm{AgNO}_{3}\right)$ 中，在 $45{ }^{\circ} \mathrm{C}$ 下反应 $5 \mathrm{~min}$ 。在此过程中, $\mathrm{Ag}$ 纳米粒子沉积在硅表面, 腐蚀后形成纳米孔。随后, 第一组硅片置于 $\mathrm{NaOH}$ 溶液中腐蚀形成纳米线面; 第二组硅片置于 $\mathrm{HF} / \mathrm{HNO}_{3}$ 混合溶液中, 将纳米孔扩 大形成微米线面; 第三组硅片在第二组的基础上再 依次置于 MCCE 溶液和 $\mathrm{NaOH}$ 溶液中，最终获得纳 米-微米的复合线面。反应后的硅片置于 $\mathrm{HNO}_{3}$ 水溶 液中去除多余的金属杂质, 得到的三种线面形貌如 图 5 所示。其中用纳米-微米线面(反射率为 15\%) 制备获得的太阳能电池片的光电转换效率可达 $18.45 \%$ 。这是由于其具有较好的表面针化, 显著减 少了载流子复合。而单纯的纳米线面虽然具有较低 的反射率(14\%), 但由于其比表面积较大, 增加了 载流子的复合, 导致太阳能电池片光电转换效率 较低。

文献[32]报道了在 Ag-MCCE 过程中 $\mathrm{Ag}^{+}$浓度对 制线形貌及电池性能的影响。随着 $\mathrm{Ag}^{+}$浓度的提高, 硅片表面会形成较深的纳米孔，可以有效提高捕获 入射光的能力。但是, 当 $\mathrm{Ag}^{+}$离子浓度达到一定值
时, 制线后晶体硅太阳能电池的性能急剧下降, 这 是由于多晶硅表面形成的孔较深, 导致表面复合中 心增多, 极大影响了其电学性能。在 $\mathrm{Ag}^{+}$的浓度为 $0.02 \mathrm{~mol} / \mathrm{L}$ 条件下制备线面结构时, 太阳能电池性 能达到最佳, 光电转换效率为 $18.94 \%$ 。

金刚线切割多晶硅片的表面存在损伤层, 因此 在 MCCE 法制线前需要将其去除。 $\mathrm{Wu}$ 等 ${ }^{[33}$ 提出了 一种通过引入人工缺陷在金刚线切割多晶硅片表面 制线的方法。该方法无需去除损伤层，直接将切割 后的多晶硅片浸入到 $\mathrm{HF} / \mathrm{HNO}_{3} / \mathrm{AgNO}_{3}$ 溶液中, 硅 片表面就会引入大量的人工缺陷。 $\mathrm{HF} / \mathrm{HNO}_{3}$ 溶液可 以将纳米级的线面腐蚀扩展为亚微米级的线面, 过 程示意图见图 6(a)。通过该方法获得的线面, 形貌 示意图和反射率曲线如图 6(b)所示，反射率约
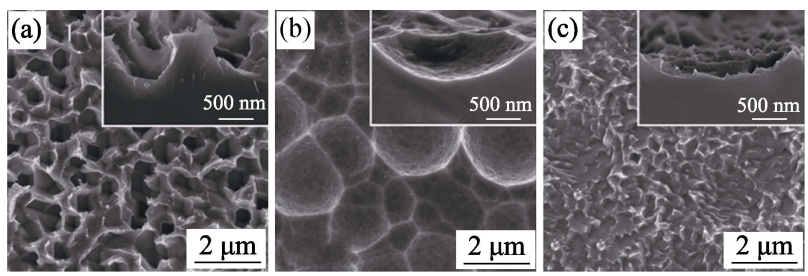

图 5 (a)纳米线面、(b)微米线面及(c)纳米-微米复合线面 SEM 表面形貌照片 ${ }^{[31]}$

Fig. 5 SEM images of silicon surfaces with (a) nano-texture, (b) micro-texture and (c) nano-micro-texture ${ }^{[31]}$ (a)

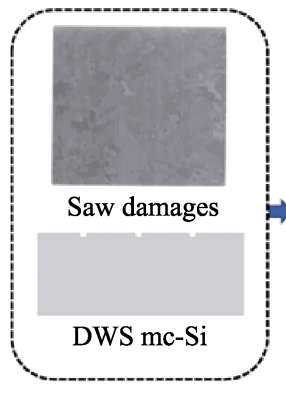

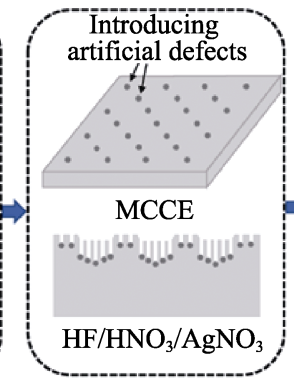
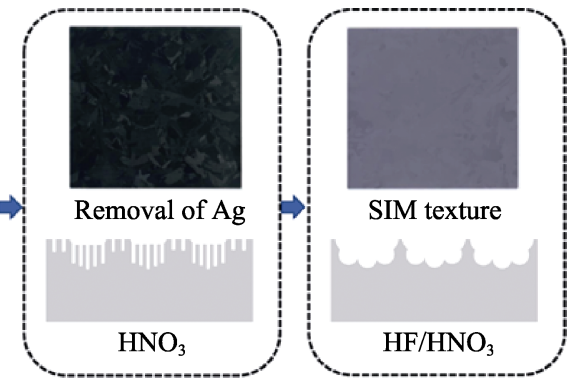

(b)

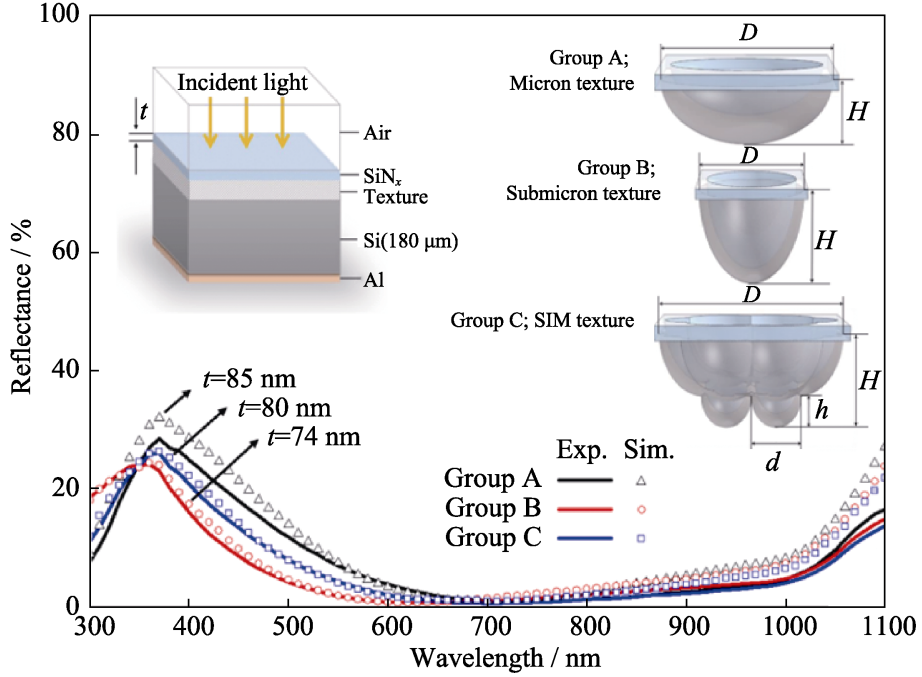

图 6 (a)DWS 多晶硅片表面制备亚微米级(SIM)线面的过程示意图和(b)三种线面结构的反射率结果 ${ }^{[33]}$

Fig. 6 (a) Schematic illustration of the main steps to prepare the submicron-in-micron (SIM) texture on the DWS mc-Si wafer and (b) experimental reflectance (curves) and simulated reflectance (scatter points) of three samples ${ }^{[33]}$ 
为 $19 \%$, 远低于传统的 $\mathrm{HF} / \mathrm{HNO}_{3}$ 制线体系。太阳能 电池的光电转换效率可以达到 $19.07 \%$ 。该方法简单 易行, 通过改进光学抗反射和表面针化, 最终提高 了电池片的光电转换效率。

碱和 MCCE 腐蚀的多晶硅均出现了各向异性腐 蚀现象, Zou 等 ${ }^{[34]}$ 结合碱腐蚀、Ag-MCCE 以及后腐 蚀处理工艺, 可以在多晶硅片表面消除各向异性腐 蚀, 获得均匀的线面结构。第一次碱腐蚀后, 在初始 的 Si(100)、(110)和(111)晶面上分别出现了倒金字 塔、阶梯以及倾斜面三种形貌。再经过 Ag-MCCE 和后腐蚀处理, 不同晶粒上的微观结构可以调整为 结构相似、反射率相近的形貌(图 7)。通过上述工艺 制备的太阳能电池片具有良好的外观和约 19.4\%的 光电转换效率。此外, 该方法获得的亚微米线面的 太阳能电池具有优良的电流-电压特性以及弱光响 应等性能。

除此之外, 文献[35-36]研究了 Ag-MCCE 制线 过程中添加剂的影响, 即在 Ag 辅助的 MCCE 法制 线过程中, 添加含有烷基酚聚氧乙烯的添加剂可以 改善 Ag 沉积, 使 Ag 颗粒均匀地沉积在硅片表面, 同时又确保颗粒大小适宜, 避免线面结构发生坍 塌。实验结果表明, 该添加剂改善了太阳能电池的 外观和性能, 获得的太阳能电池的最高光电转换效 率为 $19.51 \%$ 。此外, 在 Ag 颗粒沉积后的腐蚀环节, 加入一种含有生物酶的添加剂, 能够使金刚线切割
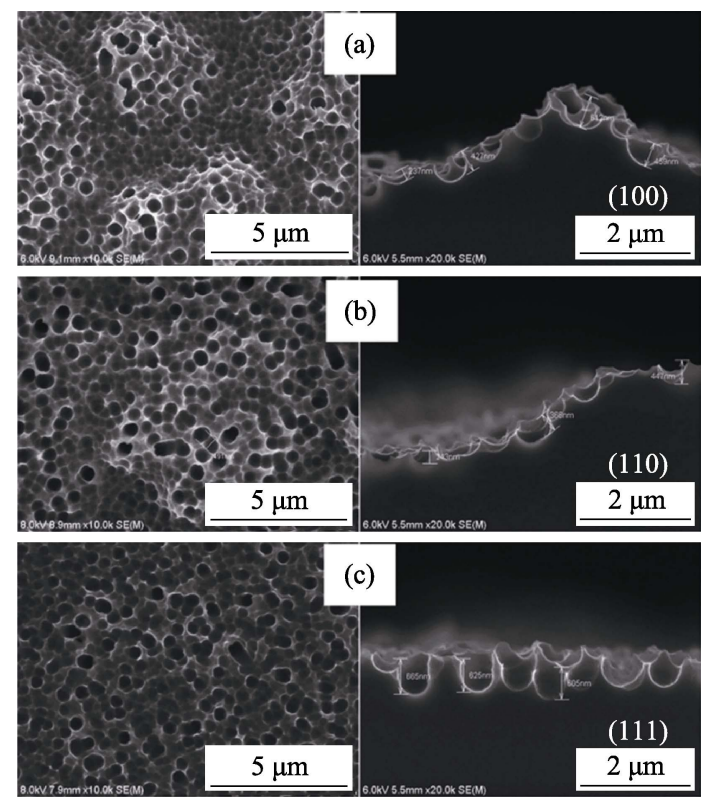

图 7 碱、Ag-MCCE 和后腐蚀处理获得的不同晶面的硅片 表面和截面 SEM 照片 ${ }^{[34]}$

Fig. 7 Surface and cross-sectional SEM images of mc-Si grains after etching by alkali, Ag-MCCE and post-etching with different orientations ${ }^{[34]}$

(a) (100); (b) (110); (c) (111)
多晶硅片表面不同取向的晶粒都获得均匀的纳米结 构, 有助于提高太阳能电池的性能, 最大光电转换 效率达到 19.56\%。并且使用添加剂后太阳能电池片 的有效少数载流子寿命和俄歇复合率分别为未使用 添加剂的两倍和三分之一。

在 Ag-MCCE 制线过程中, 目前主要采用复合 工艺, 同时引入适当的添加剂, 对制线过程进行优 化, 以获得结构和性能优良的线面, 进而改善太阳 能电池的性能。

\section{$2.2 \mathrm{Cu}$ 催化化学腐蚀}

湿法黑硅技术中主要采用的 Au、Ag、Pt 等金 属价格昂贵, 且具有较高的氧化还原电位和催化活 性, 导致在 MCCE 过程中腐蚀反应速率过快, 在硅 片表面形成大面积的纳米多孔和纳米线阵列。这往 往造成太阳能电池片针化效果差、电流收集效率低 等缺点。

针对上述问题, 近年来 $\mathrm{Cu}$ 催化剂逐渐被人们 所关注。但是不同于 $\mathrm{Ag}$ 等催化剂, $\mathrm{Cu}$ 在催化腐蚀 过程中, 随着 $\mathrm{Cu}^{2+}$ 不断被还原, 铜颗粒不断长大, 在硅片表面形成一层致密的铜膜 ${ }^{[37]}$ 。铜膜阻碍了腐 蚀液与硅表面的进一步接触, 使反应停滞, 无法在 硅片表面获得线面结构。因此, 往往需要在 Cu-MCCE 过程中加入氧化剂, 例如 $\mathrm{H}_{2} \mathrm{O}_{2}$ 或 $\mathrm{FeCl}_{3}$ 等, 持续氧化 $\mathrm{Cu}$, 以确保腐蚀反应持续进行 ${ }^{[38]}$ 。

在传统的酸腐蚀基础上，有文献进行了酸腐蚀 和铜催化腐蚀的复合制线研究。Zou 等 ${ }^{[39]}$ 采用酸性 湿法刻蚀预处理工艺, 结合低成本的 Cu-MCCE 过 程成功实现了金刚线切割多晶硅片的表面制线, 获 得结构均匀的倒金字塔线面。为了研究不同铜源对 制线过程的影响, Sheng 等 ${ }^{[40]}$ 分别选用 $\mathrm{CuSO}_{4}$ 、 $\mathrm{CuCl}_{2}$ 和 $\mathrm{Cu}\left(\mathrm{NO}_{3}\right)_{2}$ 作为铜源, 不同的阴离子会影响 溶液中电荷的传递, 影响腐蚀速率。上述三种铜源 制线后分别获得了沟槽状、蠕虫状和倒金字塔状结 构的线面。在铜催化腐蚀制线过程中, Wang 等 ${ }^{[41]}$ 采用 $\mathrm{HF} / \mathrm{HNO}_{3} / \mathrm{H}_{3} \mathrm{PO}_{4}$ 的混合溶液进行后处理, 修饰 线面结构, 形成了亚微米级的多边形雉孔状结构 (图 8)。该结构可以有效抑制表面复合，提高太阳能 电池的光电性能, 最高光电转换效率达到 $18.88 \%$ 。 Zha 等 ${ }^{[42]}$ 利用 $\mathrm{Cu}$ 催化外加 $\mathrm{HNO}_{3} / \mathrm{HF}$ 溶液腐蚀可以 形成 300 600 nm 的亚微米级线面, 制备的金刚线 切割多晶硅太阳能电池的转换效率达到 19.06\%。

\section{$2.3 \mathrm{Ag}-\mathrm{Cu}$ 复合催化化学腐蚀}

在 Ag-MCCE 过程中 Ag 颗粒沿着垂直于硅片表 面的方向沉积, 而在 Cu-MCCE 过程中, $\mathrm{Cu}$ 颗粒沿 着腐蚀孔侧壁的方向沉积。因此, 采用 $\mathrm{Ag}-\mathrm{Cu}$ 复合 

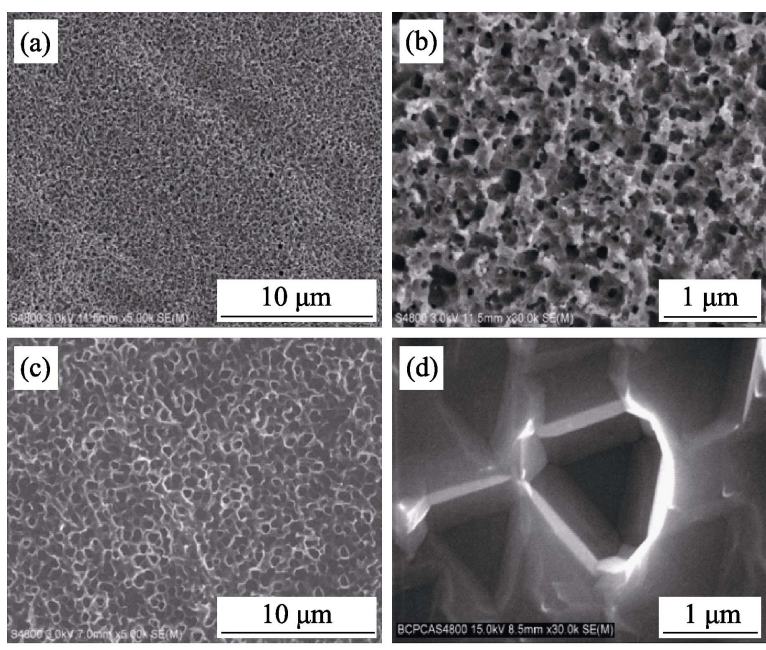

(e)

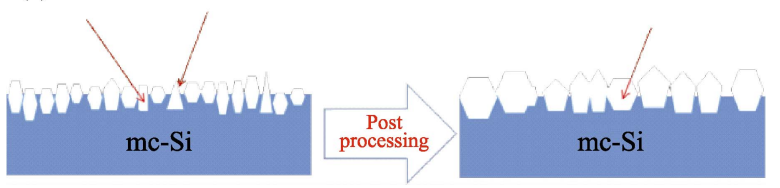

图 $8 \mathrm{Cu}-\mathrm{MCCE}$ 制线后采用后处理工艺 $(\mathrm{a}, \mathrm{b})$ 前和(c, d)后的 硅片形貌图以及(e)后处理工艺示意图 ${ }^{[41]}$

Fig. 8 SEM images of $\mathrm{Si}$ nanostructures produced by Cu-MCCE method (a,b) before and (c,d) after the post-processing treatment and (e) schematic diagram of post-processing treatment ${ }^{[41]}$

催化剂共同进行腐蚀，既可以减少贵金属 Ag 的消 耗，又可以获得均匀的线面结构。

Zheng 等 ${ }^{[43]}$ 在室温下采用一步 Ag-Cu 双原子催 化腐蚀法制备线面结构, 研究了 Ag-Cu 摩尔比对多 晶硅表面反射率及形貌的影响。通过实验发现 $\mathrm{Ag}-\mathrm{Cu}$ 双原子催化腐蚀法制备的线面结构比单独催 化更加平整，且反射率更低。Wang 等 ${ }^{[44]}$ 研究了 $\mathrm{Cu}$ 、 $\mathrm{Ag}$ 以及 $\mathrm{Ag}-\mathrm{Cu}$ 作为催化剂的金属催化腐蚀制线, 探索不同比例的 $\mathrm{Cu}\left(\mathrm{NO}_{3}\right)_{2}: \mathrm{HF} 、 \mathrm{AgNO}_{3}: \mathrm{HF}$ 和 $\mathrm{AgNO}_{3}: \mathrm{Cu}\left(\mathrm{NO}_{3}\right)_{2}$ 对线面结构的影响。研究表明, 由 于硅各晶面的原子受背成键结合力不同, 在加入不 同比例的 $\mathrm{Cu}^{2+}$ 和 $\mathrm{Ag}^{+}$时, 由于腐蚀速率的差异, 可 在不同晶面上获得雉形、楔形、倒金字塔状等不同 的线面结构。在 MCCE 制线后, 采用 $\mathrm{NaF} / \mathrm{H}_{2} \mathrm{O}_{2}$ 的 混合溶液进行后处理, 腐蚀纳米结构和去除氧化层, 获得了类似倒金字塔的结构, 硅片表面的反射率低 于传统的 $\mathrm{HF} / \mathrm{HNO}_{3}$ 酸制线体系。

Chen 等 ${ }^{[45]}$ 采用一步 Ag-Cu 共同催化腐蚀法制 备金刚线切割多晶硅片的表面线面, 研究了不同取 向的晶面在腐蚀过程中的腐蚀反应。结果表明, 由 于不同晶面硅原子的背成键结合力不同, 不同取向 上的腐蚀速率差异较大, 可制备出不同夹角的雉 形、倒金字塔状线面。该方法既不需要去除划痕，也 不需要进行后处理。在腐蚀过程中 Ag 催化腐蚀挖
孔和 $\mathrm{Cu}$ 催化腐蚀扩孔同时完成。由于倒金字塔结 构良好的减反射及钝化效果, 大大提高了太阳能电 池片的性能, 光电转换效率达 $19.49 \%$ 。如图 9 所示 为 Cu-MCCE 和 Ag-MCCE 处理后的硅片截面形貌 $\mathrm{SEM}$ 照片以及 $\mathrm{Ag}-\mathrm{Cu}$ 复合制线过程的机理示意图。

\section{4 其它金属催化化学腐蚀}

除了常用的 $\mathrm{Ag}$ 和 $\mathrm{Cu}$ 催化剂外, 还有其它金属 可用于 MCCE 法制线。有报道 ${ }^{[46]}$ 称在硅表面采用磁 控溅射法沉积 $\mathrm{Ni}$ 纳米颗粒, 然后置于 $\mathrm{HF} / \mathrm{H}_{2} \mathrm{O}_{2}$ 的混 合溶液中进行腐蚀, 可以制备出低反射率的表面结 构。Takaloo 等 ${ }^{[47]}{ }$ 研究了在金刚线切割多晶硅片表 面 $\mathrm{Ni}$ 催化一步法制线。他们选用 $\mathrm{HF} / \mathrm{NiSO}_{4} / \mathrm{H}_{2} \mathrm{O}_{2}$ 的混合溶液进行腐蚀, 通过 SEM 和电子背散射衍 射研究不同取向晶面的腐蚀过程, 最终获得了反射 率较低的纳米级多孔结构, 太阳能电池片的光电转 换效率为 $16.60 \%$ 。Gao 等 ${ }^{[48]}$ 在 Cu-MCCE 的基础上, 通过在溶液中加入 $\mathrm{Ni}$ 离子, 大大提高了 $\mathrm{Cu}-\mathrm{MCCE}$ 的制线速率，获得的线面平均反射率为 $18.53 \%$ 。

此外，有大量文献报道了在单晶硅和多晶硅表 面采用 $\mathrm{Au}^{[49-52]} 、 \mathrm{Pt}^{[53-55]} 、 \mathrm{Fe}^{[38]}$ 进行催化腐蚀制备纳 米孔或纳米线结构, 但是鲜有相关金属在金刚线切 割多晶硅片表面制线的应用报道。目前广泛采用的 MCCE 的金属仍然主要为 $\mathrm{Ag}$ 和 $\mathrm{Cu}$ 。

\section{5 不同 MCCE 法获得的线面性能对比}

表 1 归纳了采用不同金属催化剂，以及不同制 线方法获得的金刚线切割多晶硅片表面反射率及太 阳能电池片的性能。由表 1 可知, 当 $\mathrm{Ag}$ 作为催化剂 时, 经催化腐蚀制线后获得的太阳能电池片光电转 换效率最高可达 $20.89 \%$, 此时反射率为 $23.7 \%$ 。这 是由于该条件下线面结构具有较好的深宽比，同时
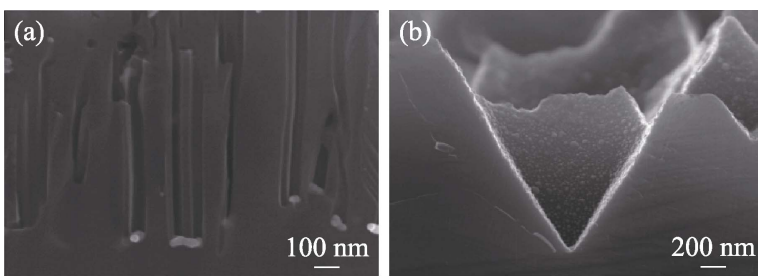

(c)

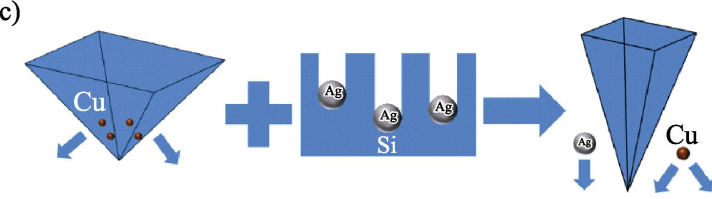

图 9 (a)Ag-MCCE 和(b)Cu-MCCE 处理后的硅片截面形貌 SEM 照片, 以及(c)单独的 Cu-MCCE、Ag-MCCE 和 Ag-Cu 复合制线过程的原理图 ${ }^{[45]}$

Fig. 9 Cross-sectional SEM images of Si wafers after (a) Ag-MCCE and (b) Cu-MCCE, and (c) schematics of etching process by single $\mathrm{Cu}$ - and Ag-catalyzed chemical etching and Ag/Cu-cocatalyzed chemical etching ${ }^{[45]}$ 
电池片表面通过沉积 $\mathrm{SiO}_{2} / \mathrm{SiN}_{x}$ 叠加层可以获得较好 的钝化效果, 经优化后最终获得较高的光电转换效 率 ${ }^{[57]}$ 。Ag 属于贵金属, 会提高制线过程的生产成本, 而且含 $\mathrm{Ag}$ 的废液较难处理。针对上述问题, 可以通 过引入适当的添加剂来减少 $\mathrm{Ag}$ 的使用, 同时提高 制线效率。此外, 引入 $\mathrm{Cu}$ 作为催化剂, 可以制备复 合线面结构, 既降低了生产成本, 也使废液更易处 理，获得的电池片光电转换效率为 $19.49 \%$ 。

图 10 为电池片光电转换效率与线面反射率之 间的关系图。由图可知, 转换效率由反射率以及线 面结构共同决定, 反射率较低可以产生更多的光生 载流子, 而线面尺寸适宜, 则可以提高表面钝化效 果, 减少载流子的复合。因此需要寻求反射率与线 面结构之间的平衡，进而提高光电转换效率。

表 2 为金刚线切割多晶硅片表面不同 MCCE 方
法的优缺点对比。从表中可知, $\mathrm{Ag}$ 和 $\mathrm{Cu}$ 仍为目前 主流的 MCCE 法制线所选用的催化剂, 具有广阔的 应用前景。这是由于 $\mathrm{Ag}$ 和 $\mathrm{Cu}$ 具有较好的催化活性,

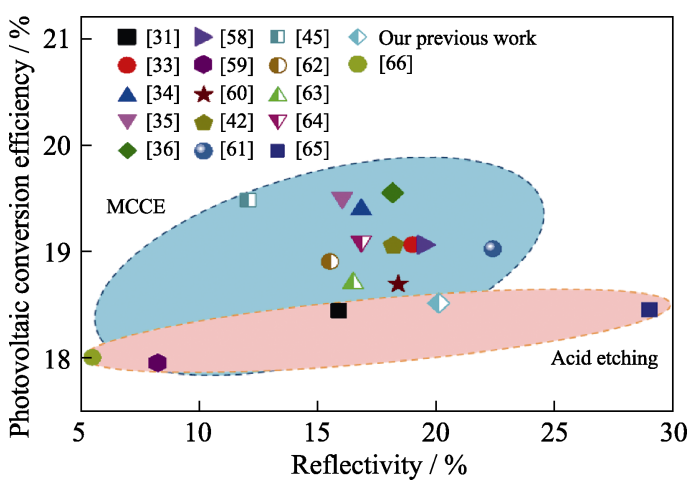

图 10 电池片光电转换效率与线面反射率之间的关系图 $[31,3336,4245,58665]$ Fig. 10 Relation between $\eta$ of solar cells and reflectivity of texture surface ${ }^{[31,33-36,42,45,58-65]}$

表 1 金刚线切割多晶硅片表面不同 MCCE 方法制线后的性能对比

Table 1 Performances for texture surfaces of DWS multicrystalline silicon prepared via different MCCE methods

\begin{tabular}{|c|c|c|c|c|}
\hline Catalyst & Method & $R^{a}$ & $\eta^{b}$ & Ref. \\
\hline $\mathrm{Ag}$ & $\mathrm{Ag}-\mathrm{MCCE}+\mathrm{HF} / \mathrm{HNO}_{3}+\mathrm{NaOH}$ & $15.9 \%$ & $18.45 \%$ & {$[31]$} \\
\hline $\mathrm{Ag}$ & Artificial defects $\left(\mathrm{HF} / \mathrm{HNO}_{3} / \mathrm{AgNO}_{3}\right)+\mathrm{HF} / \mathrm{HNO}_{3}$ & $19 \%$ & $19.07 \%$ & {$[33]$} \\
\hline $\mathrm{Ag}$ & Alkali etching+Ag-MCCE+post etching & $16.85 \%$ & $19.4 \%$ & {$[34]$} \\
\hline $\mathrm{Ag}$ & Ag deposition (additive)+etching & $16.04 \%$ & $19.51 \%$ & [35] \\
\hline $\mathrm{Ag}$ & Ag deposition+etching (additive) & $18.17 \%$ & $19.56 \%$ & {$[36]$} \\
\hline $\mathrm{Ag}$ & $\mathrm{HF} / \mathrm{HNO}_{3}+\mathrm{Ag}-\mathrm{MCCE}+\mathrm{RIE}$ & - & $20.69 \%$ & {$[56]$} \\
\hline $\mathrm{Ag}$ & Ag-MCCE & $23.7 \%$ & $20.89 \%$ & {$[57]$} \\
\hline $\mathrm{Ag}$ & Ag-MCCE+modification by acid etching & $19.46 \%$ & $19.07 \%$ & {$[58]$} \\
\hline $\mathrm{Ag}$ & $\mathrm{HF} / \mathrm{HNO}_{3}+\mathrm{Ag}-\mathrm{MCCE}+\mathrm{NSR}$ process & $8.26 \%$ & $17.96 \%$ & [59] \\
\hline $\mathrm{Ag}$ & $\mathrm{HF} / \mathrm{HNO}_{3}+\mathrm{Ag}-\mathrm{MCCE}+\mathrm{HF} / \mathrm{HNO}_{3}$ & $18.4 \%$ & $18.7 \%$ & {$[60]$} \\
\hline $\mathrm{Cu}$ & Cu-MCCE+post etching $\left(\mathrm{HF} / \mathrm{HNO}_{3} / \mathrm{H}_{3} \mathrm{PO}_{4}\right)$ & - & $18.88 \%$ & {$[41]$} \\
\hline $\mathrm{Cu}$ & $\mathrm{Cu}-\mathrm{MCCE}+\mathrm{HF} / \mathrm{HNO}_{3}$ & $18.21 \%$ & $19.06 \%$ & {$[42]$} \\
\hline $\mathrm{Cu}$ & Cu-MCCE & $22.4 \%$ & $19.03 \%$ & {$[61]$} \\
\hline Ag-Cu & Cu/Ag-MCCE & $12.08 \%$ & $19.49 \%$ & {$[45]$} \\
\hline Ag-Cu & Alkali pretreatment (additive)+Cu/Ag-MCCE+post etching & $15.52 \%$ & $18.91 \%$ & {$[62]$} \\
\hline $\mathrm{Ag}-\mathrm{Cu}$ & $\mathrm{Cu} / \mathrm{Ag}-\mathrm{MCCE}+\mathrm{NSR}\left(\mathrm{H}_{2} \mathrm{O}_{2} / \mathrm{NaF}\right)$ & $16.50 \%$ & $18.71 \%$ & {$[63]$} \\
\hline $\mathrm{Ag}-\mathrm{Cu}$ & $\mathrm{Cu} / \mathrm{Ag}-\mathrm{MCCE}+\mathrm{NSR}\left(\mathrm{H}_{2} \mathrm{O}_{2} / \mathrm{NaF}\right)$ & $16.85 \%$ & $19.10 \%$ & {$[64]$} \\
\hline $\mathrm{Ni}$ & Ni-MCCE & - & $16.60 \%$ & {$[47]$} \\
\hline $\mathrm{Cu}-\mathrm{Ni}$ & $\mathrm{Cu} / \mathrm{Ni}-\mathrm{MCCE}\left(\mathrm{Cu}\left(\mathrm{NO}_{3}\right)_{2}+\mathrm{NiSO}_{4}+\mathrm{HF}+\mathrm{H}_{2} \mathrm{O}_{2}\right)$ & $18.53 \%$ & - & [48] \\
\hline
\end{tabular}

Note: (a) $R$ represents the reflectivity of texture surface with the highest $\eta$; (b) $\eta$ represents the maximum photoelectric conversion efficiency of solar cells obtained in the literature.

表 2 金刚线切割多晶硅片表面不同 MCCE 方法的对比

Table 2 Comparison of making texture surface on DWS multicrystalline silicon by different MCCE methods

\begin{tabular}{|c|c|c|c|}
\hline Method & Advantages & Disadvantages & $\eta$ \\
\hline Ag-MCCE & $\begin{array}{l}\text { Mature technology, easily formed nanostructure, stable } \\
\text { performance }\end{array}$ & High cost, difficultly to recycled waste liquid & $20.89 \%$ \\
\hline Cu-MCCE & $\begin{array}{l}\text { Low cost, easily removed residual } \mathrm{Cu} \text {, significantly reduced } \\
\text { impact of saw marks }\end{array}$ & $\begin{array}{l}\text { Easily formed dense film, decreased etching rate, essential } \\
\text { oxidants }\end{array}$ & $19.06 \%$ \\
\hline MCCE-additive & Uniform size, stable performance & $\begin{array}{l}\text { Organic compounds increasing the cost of waste liquid } \\
\text { treatment }\end{array}$ & $19.56 \%$ \\
\hline Composite MCCE & Composite structure & $\begin{array}{l}\text { Complicated process, difficultly to recycled waste } \\
\text { liquid }\end{array}$ & $19.49 \%$ \\
\hline Other metal-MCCE & Low cost, composite structure & Inmature & $16.60 \%$ \\
\hline
\end{tabular}


可以通过在催化过程中改变腐蚀条件, 调控线面结 构。同时, 采用适当的添加剂, 可以显著提高制线的 效率及线面的结构均匀性和电池性能。既减少了金 属的消耗, 降低了生产成本, 又可以更有效地处理 制线后的废液, 减少环境污染。

\section{3 展望}

目前金刚线切割多晶硅片的主流制线技术为 MCCE 法, 其具有过程可控、表面结构均匀、减反 射效果好等优点, 具有广阔的发展应用前景, 但是 仍然存在不足，需要从以下几个方面解决:

1) 在 MCCE 法制线过程中, 不同金属颗粒在 硅中的沉积过程还不明晰，同时金属颗粒沉积的形 状及大小的影响因素也尚未明确, 缺乏系统地针对 不同晶面、不同金属颗粒对腐蚀速率、腐蚀形貌等 影响的研究。

2) 目前 MCCE 法制线为槽式制线, 相较于传 统的酸腐蚀链式制线, 具有工艺复杂, 流程较长, 时间成本和原料成本高等缺点。因此, 需要缩短制 线环节，提高制线效率，从而节约成本。

3) 目前针对 MCCE 法制线过程中添加剂对金 属的沉积、腐蚀以及扩孔过程影响的研究较少。如 何加入适当的添加剂获得结构均匀、深宽比优良且 性能良好的线面，也将是今后的研究热点。

\section{参考文献:}

[1] ADEBISI J A, AGUNSOYE J O, BELlO S A, et al. Potential of producing solar grade silicon nanoparticles from selected agro-wastes: a review. Sol. Energy, 2017, 142: 68-86.

[2] YIN Y, GAO Y, LI X, et al. Experimental study on slicing photovoltaic polycrystalline silicon with diamond wire saw. Mat. Sci. Semicon. Proc., 2020, 106: 104779.

[3] BASHER M K, MISHAN R, BISWAS S, et al. Study and analysis the $\mathrm{Cu}$ nanoparticle assisted texturization forming low reflective silicon surface for solar cell application. AIP Adv., 2019, 9: 075118.

[4] ANDREANI L C, BOZZOLA A, KOWALCZEWSKI P, et al. Silicon solar cells: toward the efficiency limits. Adv. Phys. X, 2019, 4(1): 1548305

[5] HUANG B J, ZHAO J , CHAI J Y, et al. Environmental influence assessment of China's multi-crystalline silicon (multi-Si) photovoltaic modules considering recycling process. Sol. Energy, 2017, 143: 132-141.

[6] MÖLLER H J, FUNKE C, RINIO M, et al. Multicrystalline silicon for solar cells. Thin Solid Films, 2005, 487(1): 179-187.

[7] MEINEL B, KOSCHWITZ T, BLOCKS C, et al. Comparison of diamond wire cut and silicon carbide slurry processed silicon wafer surfaces after acidic texturisation. Mat. Sci. Semicon. Proc., 2014, 26: 93-100.

[8] WU Y F, CHEN Y M. Separation of silicon and silicon carbide using an electrical field. Sep. Purif. Technol., 2009, 68(1): 70-74.

[9] HARDIN C W, QU J, SHIH A J. Fixed abrasive diamond wire saw slicing of single-crystal silicon carbide wafers. Mater. Manuf. Processes, 2004, 19(2): 355-367.

[10] QIU M B, HUANG Y H, LIU Z D, et al. A review of the fabrication methods for solar silicon wafer. Mechanical Science and Technology for Aerospace Engineering, 2008, 27(8): 1017-1020.

[11] CAI E, TANG B, FAHRNER W R, et al. Characterization of the Surfaces Generated by Diamond Cutting of Crystalline Silicon. 26th European Photovoltaic Solar Energy Conference and Exhibition. Hamburg, 2011: 1884-1886.

[12] CHEN C C A, CHAO P H. Surface texture analysis of fixed and free abrasive machining of silicon substrates for solar cell. Adv. Mater. Res., 2010, 126-128: 177-180.

[13] BIDIVILLE A, WASMER K, KRAFT R, et al. Diamond Wire-sawn Silicon Wafers-from the Lab to the Cell Production. 24th European Photovoltaic Solar Energy Conference and Exhibition. Hamburg, 2009: 1400-1405.

[14] LIPPOLD M, BUCHHOLZ F, GONDEK C, et al. Texturing of $\mathrm{SiC}$-slurry and diamond wire sawn silicon wafers by $\mathrm{HF}-\mathrm{HNO}_{3}-\mathrm{H}_{2} \mathrm{SO}_{4}$ mixtures. Sol. Energ. Mat. Sol. C, 2014, 127: 104-110.

[15] MEMMING R, SCHWANDT G. Anodic dissolution of silicon in hydrofluoric acid solutions. Surf. Sci., 1966, 4(2): 109-124

[16] XIAO Z G, GENG G Y, WEI X Q, et al. On the mechanism of the vapor etching of diamond wire sawn multi-crystalline silicon wafers for texturing. Mat. Sci. Semicon. Proc., 2016, 53: 8-12.

[17] JANSEN H, DEBOER M, LEGTENBERG R, et al. The black silicon method-a universal method for determining the parameter setting of a fluorine-based reactive ion etcher in deep silicon trench etching with profile control. J. Micromech. Microeng., 1995, 5(2): 115-120.

[18] HUANG Z P, GEYER N, WERNER P, et al. Metal-assisted chemical etching of silicon: a review. Adv. Mater., 2011, 23(2): 285-308.

[19] HSU C, WU J R, LU Y T, et al. Fabrication and characteristics of black silicon for solar cell applications: an overview. Mat. Sci. Semicon. Proc., 2014, 25: 2-17.

[20] DIMOVA-MALINOVSKA D, SENDOVA-VASSILEVA M, TZENOV N, et al. Preparation of thin porous silicon layers by stain etching. Thin Solid Films, 1997, 297(1/2): 9-12.

[21] LI X, BOHN P W. Metal-assisted chemical etching in $\mathrm{HF} / \mathrm{H}_{2} \mathrm{O}_{2}$ produces porous silicon. Appl. Phys. Lett., 2000, 77(16): 2572-2574.

[22] HUANG Z P, WU Y, FANG H, et al. Large-scale $\mathrm{Si}_{1-x} \mathrm{Ge}_{x}$ quantum dot arrays fabricated by templated catalytic etching. Nanotechnology, 2006, 17(5): 1476-1480.

[23] HUANG Z, SHIMIZU T, SENZ S, et al. Ordered arrays of vertically aligned [110] silicon nanowires by suppressing the crystallographically preferred $<100>$ etching directions. Nano Lett. 2009, 9(7): 2519-2525.

[24] PENG K Q, HU J J, YAN Y J, et al. Fabrication of single-crystalline silicon nanowires by scratching a silicon surface with catalytic metal particles. Adv. Funct. Mater., 2006, 16(3): 387-394.

[25] CHATTOPADHYAY S, BOHN P W. Direct-write patterning of microstructured porous silicon arrays by focused-ion-beam $\mathrm{Pt}$ deposition and metal-assisted electroless etching. J. Appl. Phys., 2004, 96(11): 6888-6894.

[26] NIU Y C, LIU H T, LIU X J, et al. Study on nano-pores enlargement during Ag-assisted electroless etching of diamond wire sawn polycrystalline silicon wafers. Mat. Sci. Semicon. Proc., 2016, 56: 119-126

[27] SU G Y, DAI X W, SUN H C, et al. The study of the defect removal etching of black silicon for diamond wire sawn multi-crystalline silicon solar cells. Sol. Energy, 2018, 170: 95-101.

[28] CAO F, CHEN K X, ZHANG J J, et al. Next-generation multi-crystalline silicon solar cells: diamond-wire sawing, nano-texture and high efficiency. Sol. Energ. Mat. Sol. C, 2015, 
141:132-138.

[29] KUMAGAI A. Texturization using metal catalyst wet chemical etching for multicrystalline diamond wire sawn wafer. Sol. Energy Mat. Sol. C, 2015, 133: 216-222.

[30] PENG K, FANG H, HU J, et al. Metal-particle-induced, highly localized site-specific etching of $\mathrm{Si}$ and formation of single-crystalline Si nanowires in aqueous fluoride solution. Chemistry, 2006, 12(30): 7942-7947.

[31] ZHUANG Y F, ZHONG S H, HUANG Z G, et al. Versatile strategies for improving the performance of diamond wire sawn mc-Si solar cells. Sol. Energ. Mat. Sol. C, 2016, 153: 18-24.

[32] ZHANG P F, JIA R, TAO K, et al. The influence of Ag-ion concentration on the performance of mc-Si silicon solar cells textured by metal assisted chemical etching (MACE) method. Sol. Energ. Mat. Sol. C, 2019, 200: 109983.

[33] WU C K, ZOU S, ZHU J Y, et al. Forming submicron in micron texture on the diamond-wire-sawn mc-Si wafer by introducing artificial defects. Prog. Photovoltaics, 2020, 28: 788-797.

[34] ZOU S, YE X Y, WU C K, et al. Complementary etching behavior of alkali, metal-catalyzed chemical, and post-etching of multicrystalline silicon wafers. Prog. Photovoltaics, 2019, 27(6): 511-519.

[35] LI X P, TAO K, ZHANG D, et al. Development of additive-assisted Ag-MACE for multicrystalline black Si solar cells. Electrochem. Commun., 2020, 113: 106686.

[36] LI X P, GAO Z B, ZHANG D, et al. High-efficiency multi-crystalline black silicon solar cells achieved by additive assisted Ag-MACE. Sol. Energy, 2020, 195: 176-184.

[37] PENG K Q, ZHU J. Morphological selection of electroless metal deposits on silicon in aqueous fluoride solution. Electrochim Acta, 2004, 49(16): 2563-2568.

[38] CAO M, LI S Y, DENG J X, et al. Texturing a pyramid-like structure on a silicon surface via the synergetic effect of copper and Fe(III) in hydrofluoric acid solution. Appl Surf. Sci., 2016, 372: 36-41.

[39] ZOU Y X, XI F S, QIU J J, et al. Cu-assisted chemical etching of diamond wire sawn multicrystalline silicon wafers for texturing. China Surface Engineering, 2017, 30(6): 59-66.

[40] SHENG G Z, ZOU Y X, LI S Y, et al. Controllable nano-texturing of diamond wire sawing polysilicon wafers through low-cost copper catalyzed chemical etching. Mater. Lett., 2018, 221: 85-88.

[41] WANG P, XIAO S Q, JIA R, et al. 18.88\%-efficient multi-crystalline silicon solar cells by combining Cu-catalyzed chemical etching and post-treatment process. Sol Energy, 2018, 169: 153-158.

[42] ZHA J W, WANG T, PAN C F, et al. Constructing submicron textures on mc-Si solar cells via copper-catalyzed chemical etching. Appl. Phys. Lett., 2017, 110(9): 093901.

[43] ZHENG C F, SHEN H L, PU T, et al. Fabrication and property of anti-reflection structures on multicrystalline silicon by $\mathrm{Ag}$ and $\mathrm{Cu}$ dually assisted chemical etching. J. Funct. Mater., 2017, 48(1): 1230-1235.

[44] WANG S D, CHEN T W. Texturization of diamond-wire-sawn multicrystalline silicon wafer using $\mathrm{Cu}, \mathrm{Ag}$, or $\mathrm{Ag} / \mathrm{Cu}$ as a metal catalyst. Appl. Surf. Sci., 2018, 444: 530-541.

[45] CHEN W, LIU Y P, WU J T, et al. High-efficient solar cells textured by $\mathrm{Cu} / \mathrm{Ag}$-cocatalyzed chemical etching on diamond wire sawing multicrystalline silicon. ACS Appl. Mater. Inter., 2019, 11(10): 10052-10058.

[46] YUE Z H, SHEN H L, JIANG Y, et al. Novel and low reflective silicon surface fabricated by Ni-assisted electroless etching and coated with atomic layer deposited $\mathrm{Al}_{2} \mathrm{O}_{3}$ film. Appl. Phys. A-Mater., 2013, 114(3): 813-817.

[47] TAKALOO A V, ES F, BAYTEMIR G, et al. Nickel assisted chemical etching for multi-crystalline Si solar cell texturing: a low cost single step alternative to existing methods. Mater. Res.
Express, 2018, 5(7): 075506.

[48] GAO K, SHEN H L, LIU Y W, et al. Enhanced etching rate of black silicon by $\mathrm{Cu} / \mathrm{Ni}$ Co-assisted chemical etching process. Mat. Sci. Semicon. Proc., 2018, 88: 250-255.

[49] LAI R A, HYMEL T M, NARASIMHAN V K, et al. Schottky barrier catalysis mechanism in metal-assisted chemical etching of silicon. ACS Appl. Mater. Inter., 2016, 8(14): 8875-8879.

[50] MAURYA M R, TOUTAM V, SINGH P, et al. Optimization of electroless plating of gold during MACE for through etching of silicon wafer. Mat. Sci. Semicon. Proc., 2019, 100: 140-144.

[51] XIE T, SCHMIDT V, PIPPEL E, et al. Gold-enhanced low-temperature oxidation of silicon nanowires. Small, 2008, 4(1): 64-68.

[52] BAYTEMIR G, CIFTPINAR E H, TURAN R. Enhanced metal assisted etching method for high aspect ratio microstructures: applications in silicon micropillar array solar cells. Sol. Energy, 2019, 194: 148-155.

[53] YAE S, KAWAMOTO Y, TANAKA H, et al. Formation of porous silicon by metal particle enhanced chemical etching in HF solution and its application for efficient solar cells. Electrochem. Commun., 2003, 5(8): 632-636.

[54] CHATTOPADHYAY S, LI X, BOHN P W. In-plane control of morphology and tunable photoluminescence in porous silicon produced by metal-assisted electroless chemical etching. J. Appl. Phys., 2002, 91(9): 6134-6140.

[55] ZHU B, LIU W J, DING S J, et al. Formation mechanism of heavily doped silicon mesopores induced by Pt nanoparticle-assisted chemical etching. J. Phys. Chem. C, 2018, 122(37): 21537-21542.

[56] JIN J, SHEN H, ZHENG P, et al. >20.5\% diamond wire sawn multicrystalline silicon solar cells with maskless inverted pyramid like texturing. IEEE J. Photovolt., 2017, 7(5): 1264-1269.

[57] SHENG J, WANG W, YE Q H, et al. MACE texture optimization for mass production of high-efficiency multi-crystalline cell and module. IEEE J Photovolt., 2019, 9(3): 918-925.

[58] SU G Y, JIA R, DAI X W, et al. The influence of black silicon morphology modification by acid etching to the properties of diamond wire sawn multicrystalline silicon solar cells. IEEE $J$. Photovolt., 2018, 8(4): 937-942.

[59] JIANG Y, SHEN H L, PU T, et al. Hybrid process for texturization of diamond wire sawn multicrystalline silicon solar cell. Phys. Status Solidi-R, 2016, 10(12): 870-873.

[60] CHEN K X, ZHA J W, HU F Q, et al. MACE nano-texture process applicable for both single- and multi-crystalline diamond-wire sawn Si solar cells. Sol. Energ. Mat. Sol. C, 2019, 191: 1-8.

[61] WU J T, LIU Y P, CHEN Q S, et al. The orientation and optical properties of inverted-pyramid-like structures on multi-crystalline silicon textured by Cu-assisted chemical etching. Sol. Energy, 2018, 171: 675-680.

[62] ZHANG P F, SUN H C, TAO K, et al. An 18.9\% efficient black silicon solar cell achieved through control of pretreatment of Ag/Cu MACE. J. Mater. Sci. -Mater. El., 2019, 30(9): 8667-8675.

[63] ZHENG C F, SHEN H L, PU T, et al. High-efficient solar cells by the $\mathrm{Ag} / \mathrm{Cu}$-assisted chemical etching process on diamond-wire-sawn multicrystalline silicon. IEEE J. Photovolt., 2017, 7(1): 153-156.

[64] SHEN H L, JIANG Y. Investigation on multi-crystalline black silicon and high efficiency solar cell based on inverted pyramid antireflective structure. Journal of Nanjing University of Aeronautics \& Astronautics, 2017, 49(5): 744-752.

[65] SREEJITH K P, SHARMA A K, KUMBHAR S, et al. An additive-free non-metallic energy efficient industrial texturization process for diamond wire sawn multicrystalline silicon wafers. Sol. Energy, 2019, 184: 162-172.

[66] LIU Y B, ZHANG J N, WANG L, et al. An innovative light trapping structure fabrication method on diamond-wire-sawing multi-crystalline silicon wafers. Chemistry Select, 2018, 3(26): 7561-7564. 